\title{
Montaigne et Shakespeare : rencontres au féminin
}

\section{Ann Moss}

\section{(2) OpenEdition \\ Journals}

Édition électronique

URL : http://journals.openedition.org/shakespeare/673

DOI : $10.4000 /$ shakespeare.673

ISSN : 2271-6424

Éditeur

Société Française Shakespeare

\section{Édition imprimée}

Date de publication : 1 novembre 2004

Pagination : 209-219

ISBN : 2-9521475-0-7

\section{Référence électronique}




\section{Shakespeare et Montaigne vers un nouvel humanisme}

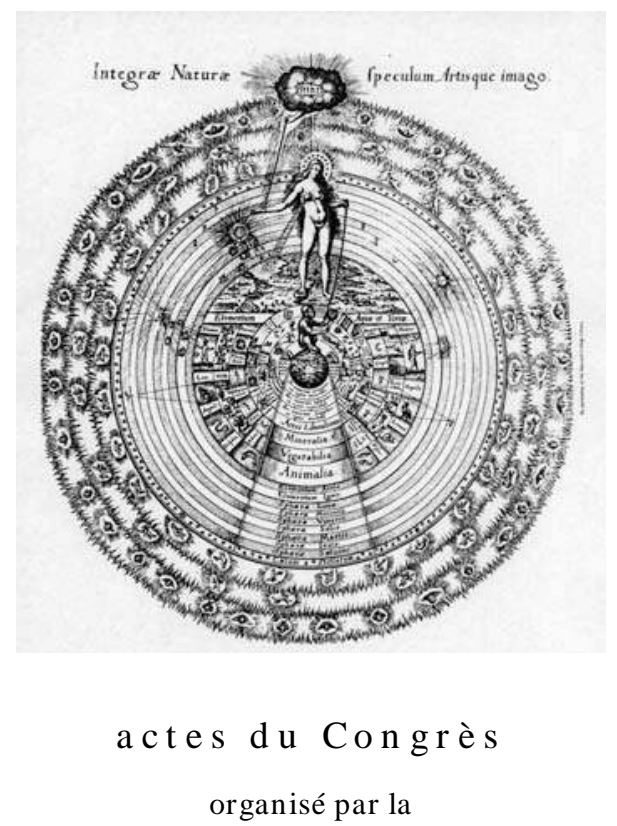

SOCIÉTÉ FR ANÇAISE SH AKESPEARE en collaboration avec la

S OC I É T É I N T E R N A T I O N A L E DES AM I D E M O N A I G N les 13,14 et 15 mars 2003

$$
\begin{gathered}
\text { textes réunis par } \\
\text { Pierre KAPITANIAK } \\
\text { sous la direction de } \\
\text { Jean-Marie MAGUIN }
\end{gathered}
$$




\section{COMITÉ SCIENTIFIQUE :}

Margaret Jones-Davis

Gisèle Venet

Jean-Marie Maguin

Yves Peyré

François Laroque

Pierre Kapitaniak

\section{COUVERTURE :}

Robert Fludd

Utriusque Cosmi Historia (1617-19)

planche 17

conception graphique et logo

Pierre Kapitaniak

\section{(C) 2003 Société Française Shakespeare}

Institut du Monde Anglophone

Université de Paris III - Sorbonne Nouvelle

http:// univ-montp3.fr/SFS/

5 rue de l'École de Médecine

75006 Paris

Diffusion :

AVL DIFFUSION

Parc Euromédecine

34198 MONTPELLIER CEDEX 5

ISBN 2-9521475-0-7

Tous droits de traduction, de reproduction et d'adaptation réservés pour tous les pays 


\title{
MONTAIGNE ET SHAKESPEARE : RENCONTRES AU FÉMININ
}

Ann Moss

\begin{abstract}
Je propose une lecture de nos auteurs axée sur les portraits de femmes mariées dont la vie (ou plutôt la mort) imite l'héroïsme stoïque de leurs maris; sur les femmes adultères moulées sur le patron des grandes séductrices, Vénus et autres; et sur le mariage vu comme symbole de la concordance entre parties opposées qui sera le grand thème des dernières œuvres des deux écrivains. Pour que ces comparaisons ne semblent qu'un catalogue de rencontres fortuites, je découvrirai le fonds de culture commun où nos deux auteurs ont puisé, la littérature gréco-romaine et les formes d'expression façonnées par l'enseignement rhétorique, pour montrer finalement comment une même mentalité se trouve à l'origine de quelques analogies entre les visions en apparence si personnelles qui seront les leurs au déclin de la vie.

Montaigne, Shakespeare, and the Feminine A survey of some aspects of the 'feminine', as it is constructed and explored in the work of our two authors. Topics to be broached: married woman imitating the heroic lifestyle (or death-style) of stoic husbands; adulterous women modelled on the Venus-type of the voluptuous female; marriage seen as a symbol of harmony between discordant parties (especially in the later works of the two writers). In order to make such comparisons seem other than merely accidental, the paper will consider them in the context of the classical culture and rhetorical theories of composition that our two writers had in common. At a deeper level of personal experience, it may be that both writers do discover a similar vision at the end of their writing careers, however different they are in important respects.
\end{abstract}

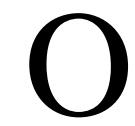

$n$ ne peut guère se mettre à faire la comparaison entre Montaigne et Shakespeare du côté des femmes sans constater que la partie est inégale ${ }^{1}$. Dans l'œuvre de Shakespeare, si fortement empreinte de l'imaginaire amoureux et passionnel de la Renaissance, on se délecte sous l'ombre des jeunes filles en fleurs, on pleure à chaudes larmes les victimes d'un sort inique, on frémit au passage de femmes mûres vouées à l'exercice d'une cruauté diabolique ou d'un érotisme exorbitant. Par contre, les femmes interviennent assez rarement dans les Essais de Montaigne, et ont bien du mal à se défaire du mauvais rôle que leur assigne cet écrivain imbu de la misogynie habituelle des écoles des humanistes où les femmes sont mises en exemple le plus souvent sous le titre de la faiblesse, du mensonge, et du manque de foi. Il n'y en a guère de bonnes dans les recueils de lieux communs issus de l'enseignement humaniste qui sont comme des abrégés de l'univers moral de l'époque et dont les traces se

\footnotetext{
${ }^{1}$ Toutes les citations de Montaigne sont prises dans l'édition des Euvres complètes établie par A. Thibaudet et M. Rat (Paris, Gallimard, 1962) ; pour les citations de Shakespeare, voir Julius Caesar dans le 'Arden Shakespeare', édité par T. S. Dorsch (Londres et New York, Methuen, 1955), et Antony and Cleopatra, aussi dans le 'Arden Shakespeare', édité par M. R. Ridley (Londres et New York, Methuen, 1954).
} 
retrouvent partout dans les Essais. Néanmoins, je peux vous en proposer trois. «Il n'en est pas à douzaines », comme dit Montaigne au début de son chapitre De trois bonnes femmes ${ }^{2}$. Et pour compenser un peu la surabondance féminine chez Shakespeare je propose de n'en choisir qu'une seule dans l'œuvre du dramaturge.

Le chapitre De trois bonnes femmes (II, 35) se met sous l'enseigne du mariage, "marché plein de tant d'espineuses circonstances », où les femmes se comportent plutôt mal du vivant de leur mari pour jouer les veuves dolentes au moment de sa mort (p.722). De prime abord, on ne s'attend pas à ce que ce scepticisme acerbe soit atténué par les récits qui suivent, qui racontent comment trois femmes ont «aussi employé l'effort de leur bonté et affection autour de la mort de leurs maris ». Pourtant, ce sont en vérité des exemples, comme dit Montaigne, « un peu autres» (p. 723). Nous y entrevoyons la possibilité d'un changement radical dans le comportement communément attribué aux femmes mariées, changement qui va jusqu'à inverser les rôles traditionnels des deux sexes. Dans les deux premiers cas, les femmes montrent la voie à leurs maris émasculés qui n'osent pas affronter une mort inévitable (dans l'un des cas cette émasculation s'entend au sens littéral puisque le mari est «merveilleusement tourmenté de quelques ulceres qui luy estoient survenus ès parties honteuses», p. 723). Les femmes viennent à l'appui des maris en agissant avec cette énergique fermeté qui depuis l'antiquité romaine est devenue l'apanage ordinaire des héros de la morale des stoïciens. L'une s'attache étroitement à son mari pour qu'ils se précipitent ensemble dans la mer, l'autre se donne la mort par un coup de poignard afin d'encourager son mari à faire de même, lui disant « Fais comme moi ; tu vois, ça ne me fait pas mal!» (p. 723, 725).

Les récits sont de bon aloi, pris chez Pline le Jeune dans ses épîtres, où il loue le courage viril des deux femmes tout en racontant assez succinctement leurs histoires ${ }^{3}$. Qu'en fait Montaigne? À la première femme, muette chez Pline ainsi que son mari, Montaigne donne une voix. C'est donc par la parole, aussi bien que par son acte, que cette femme l'emporte sur son mari. Et que dit-elle ? Elle parle du «plaisir» de se délivrer des tourments tous les deux en même temps, du bonheur de s'en aller ensemble, et lorsque Montaigne la décrit en

${ }^{2}$ Le chapitre se trouve aux pages 722-9 de l'édition citée.

${ }^{3}$ Pline le Jeune, Epistulae, vI, xxiv et III, xvi. 
train de se lier à son mari pour ce suicide à deux, il constate, ce que ne fait pas Pline, que c'est pour mourir «entre ses bras», toute pleine de «cette loyale et vehemente affection dequoy elle l'avoit embrassé pendant sa vie» (p. 723). Même chose pour la femme du second récit, où Montaigne renchérit sur le texte original lorsqu'il embellit «cette haute et courageuse entreprinse » de se poignarder pour donner de la force à son mari, en faisant remarquer qu'elle «ne regarde qu'à luy encores au dernier trait de sa vie, et à luy oster la crainte de la suivre en mourant» (p.725). Ces deux femmes s'attirent les louanges dues à ceux qui se moulent au patron de l'héroïsme très mâle associé à l'éthique des stoïciens. Mais leur exemplarité est pourtant «un peu autre». Au moment d'affronter courageusement la mort, elles se conduisent aussi en femmes, elles se consacrent à l'amour de leurs maris plutôt qu'à leur propre gloire. C'est ainsi que Montaigne trouve le moyen de placer ces récits sous la rubrique du mariage annoncé au début du chapitre, bien qu'ils aillent à l'encontre de ses opinions pour lors sceptiques sur ce sujet.

Son troisième récit va dans le même sens, mais il va plus loin. Le héros en est l'incarnation même du stoïcisme, Sénèque au moment de son suicide forcé, dont Montaigne dépeint toute la noblesse et tous les détails pitoyables qu'il avait trouvés dans les Annales de Tacite. ${ }^{4}$ Mais encore une fois, c'est un suicide à deux. La femme de Sénèque, Pauline, en demande sa part, et à vive voix chez Montaigne, tandis que chez Tacite elle joue un rôle plus réduit et sans rien dire. Pressée par son mari de continuer à vivre et de ne se montrer forte qu'en endurance morale, la Pauline de Montaigne rejette fièrement cette passivité et réclame son droit à l'action héroïque. Elle aussi sait «bien mourir ». Mais, comme dans les cas précédents, Montaigne renforce l'acte viril d'affronter la mort auquel se décide cette femme en faisant valoir que c'est par l'amour qui l'y pousse aussi. "La magnanimité de son courage » est «reschauffée » par « une très-noble affection », à laquelle Sénèque est sensible : «la constance et la resolution», dit-il, « soyent pareilles à notre commune fin, mais la beauté et la gloire soit plus grande de ta part» (p. 727). En fait, Pauline manque son suicide, elle doit survivre à son mari et traîner une vie pénible, mais il en résulte que d'une certaine façon elle devient héroïne à trois comptes :

${ }^{4}$ Tacite, Annales, XV, lxi-lxiii. 
hérö̈ne en «vertu» masculine, hérö̈ne en patience plutôt féminine, héroïne d'amour.

En faisant agir ses trois bonnes femmes selon le code stoïcien, Montaigne leur prête un caractère masculin, de même que ses sources antiques. Mais lorsqu'il s'écarte de ses auteurs pour souligner la part que joue l'amour conjugal, la «bonté » et «affection», dans la mâle résolution de ces femmes, Montaigne semble leur restituer une féminité qui en fait des héros nouveaux, ambigus, voire androgynes. On dirait des personnages de roman, peut-être des personnages de théâtre. Montaigne fait lui-même la liaison entre ses « trois contes trèsveritables » et une production littéraire où l'histoire proprement dite se transforme aisément en contes «plaisans et tragiques» (p.728). Il semble que les héroïnes mâles mais passionnelles qu'il configure dans ce chapitre soient justement le lieu de rencontre entre la prose tant soit peu revêche d'un Pline ou d'un Tacite et une poésie consacrée aux fables plaisantes et tragiques. Ses modèles préférés pour enchaîner de telles narrations seraient les Métamorphoses d'Ovide et le long poème $\mathrm{d}^{\prime}$ Arioste ${ }^{5}$. Nous pourrions y ajouter peut-être les Histoires tragiques dont s'inspirera Shakespeare.

Le chapitre de Montaigne nous offre aussi une nouvelle perspective sur le mariage. Il avait débuté sur le ton sceptique. En passant par les trois exemples de dévouement marital, nous en sommes arrivés à Sénèque, source de la morale stoïcienne. Et c'est en citant un assez long passage d'une lettre de Sénèque que Montaigne termine son chapitre sur une analyse attendrissante de l'amour de ce vieillard pour sa jeune femme qui l'incite à «m'aymer moy-mesme plus curieusement », qui le détourne de l'idée fixe de braver constamment la mort, qui l'invite à veiller sur sa santé, à conserver sa vieillesse $(\text { p. } 729)^{6}$. Cette page sortie inopinément des mains du grand stoïcien à la mort héroïque duquel nous venons d'assister associe l'amour conjugal à l'intérêt que peut prendre l'intellect à cultiver 'curieusement' le bien-être du corps. Elle trouvera l'approbation de Montaigne luimême vieillissant. Ces mots de Sénèque sont «excellans» ajoutera-t-il au moment de revoir son chapitre vers la fin de sa vie. Nous reviendrons brièvement à ce sujet.

${ }^{5}$ Montaigne a éliminé la référence à Arioste pour l'édition posthume, signe peut-être de son dédain affecté pour les contes fantastiques des modernes.

${ }^{6}$ Montaigne donne une traduction fidèle du passage de Sénèque, Epistulae, cIv, 1-5. 
Le chapitre De trois bonnes femmes fait pendant au chapitre suivant Des plus excellens hommes (II, 36), qui sont aussi au nombre de trois: Homère, Alexandre, Épaminondas. Si l'admiration de Montaigne à l'égard d'Homère reste constante, les éloges qu'il offre de prime abord aux grands hommes politiques et militaires, Alexandre et Épaminondas, se rendent de plus en plus équivoques à mesure qu'il élabore son texte au cours des années. Dans le cas des héros traditionnels, dont la gloire consiste à manier les affaires publiques et à se battre courageusement, le scepticisme de Montaigne va toujours croissant. En revanche, dans le cas de ses femmes mariées, nous avons vu céder son scepticisme à l'évidence de leur «bonté et affection». Dans Julius Caesar de Shakespeare, nous verrons sur la même scène un homme qui s'exerce à mettre en pratique la moralité rigoureuse des stoïciens et sa femme, son émule, qui deviendra son précepteur.

Cette pièce de Shakespeare est un lieu d'essai. Nous y voyons Portia et Brutus s'essayer au rôle du héros fier d'affronter les dangers et la mort avec la constance mâle de ceux qui « love The name of honour more than [they] fear death» (Julius Caesar, I.ii.87-8). Mais ce courage monumental, éminemment romain, s'érige sur un terrain peu stable. Dès la première scène du deuxième acte, nous voyons les crevasses prêtes à s'ouvrir sur le doute, la mauvaise conscience, et l'hypocrisie qui se déguise même à soi. Cette scène nous retire des places publiques et des rues de la ville si peuplées du premier acte pour nous introduire dans l'intimité de la vie domestique. Brutus, troublé, insomniaque, se promène dans son jardin. C'est là, dans ce lieu privé, qu'il ressent le déchirement douloureux d'une âme suspendue entre la notion de l'acte à accomplir et le premier mouvement qui déclenchera cet acte. C'est là, entouré d'un auditoire composé des conspirateurs, qu'il s'engage dans le complot contre Jules César, mais en se persuadant que le meurtre brutal à venir sera un sacrifice quasi rituel, une offrande aux dieux, une purgation. Ce qui lui fait horreur, c'est le sang qu'il faudra verser: «But, alas, Caesar must bleed for it » (II.i.170-1). Et en effet un filet rouge de sang laissera des traces indélébiles tout au long de la pièce. Mais Brutus a volontairement assumé son rôle de héros, de personnage énergique et constant, bien que nous comprenions que c'est un acteur masqué qui parle : 
Let not our looks put on our purposes,

But bear it as our Roman actors do,

With untir'd spirits and formal constancy

Seulement nous ne sommes plus sur les tréteaux de la place publique du premier acte. Nous sommes dans la maison conjugale. Et voici la femme de Brutus, Portia, qui vient pour tendre à son mari un tout autre portrait de lui-même que celui que Cassius lui faisait voir dans le miroir fascinant de la tentation héroïque ou que Brutus vient de composer pour son rôle de sacrificateur et le masque qu'il assume hypocritement ${ }^{7}$. Brutus est devenu depuis peu un mari négligent, impatient, préoccupé, toujours en colère. Dans l'intimité du mariage, il ne porte plus de masque, il est faible, il est inconstant. Et c'est sa femme qui sait déchiffrer ces signes extérieurs pour pénétrer jusqu'à la vérité interne: "You have some sick offence within your mind » (II.i.268). Elle le comprend si bien parce que le mariage l'a paradoxalement dévêtue de son statut de femme, elle est incorporée à Brutus, elle est sa moitié, «[his] self» (II.i.274). Biologiquement elle reste femme, «I grant I am a woman », phrase qu'elle répète à deux reprises (II.i.292, 294), mais son sang aussi bien que son mariage attestent son droit à une masculinité d'esprit et de cœur. Car, épouse de Brutus, elle est aussi fille de Caton :

Think you I am no stronger than my sex,

Being so father'd and so husbanded?

Et pour prouver son droit de cité dans cette compagnie d'hommes forts, elle s'est blessée à la cuisse, façon oblique peut-être de substituer au sexe naturel une fente faite volontairement pour se pénétrer. Au moins dit-elle avoir «made strong proof of my constancy» (II.i.299), ce que Brutus, malgré toutes ses belles paroles de parade, tarde encore à faire.

Sous cet aspect, Portia ressemble fort aux bonnes femmes de Montaigne, celles qui savent montrer à leurs maris le chemin de la gloire masculine. Brutus la reconnaît comme telle : «O ye gods, Render me worthy of this noble wife!» (II.i.302-3). Et il y a plus. Shakespeare renchérit sur le récit de Plutarque dans sa Vie de Brutus qu'il suit de près en introduisant toute la tendresse d'un mariage entre gens qui s'aiment, tout ce qui fait que Brutus compare cette estimable femme à

${ }^{7}$ Pour le miroir de Cassius, voir I, 50-69. 
ce sang qui lui est si précieux qu'il ne voudrait pas le dérober même à son ennemi :

You are my true and honourable wife,

As dear to me as are the ruddy drops

That visit my sad heart.

Mais dans cette pièce si romaine, les femmes sont interdites dans les lieux publics. Portia plus tard comprendra qu'elle ne pourra pas suivre son mari dans la rue qui mène au Capitole (II.iv). Elle doit garder la maison, où elle est vaincue momentanément par une faiblesse et une vacillation de femme privée de sa «moitié », son «self», qui lui assurait sa force. Dans sa mort, néanmoins, après son suicide de femme délaissée et désespérée, elle sera l'inspiration secrète qui décide son mari à se comporter avec une constance de philosophe stoïcien : «No man bears sorrow better. Portia is dead [...] I have the patience to endure it now » (IV.iii.146, 191). Dans son suicide à lui, Brutus s'avérera enfin «worthy of this noble wife », digne de cette femme si noble.

L'antiquité, à travers les pages de Pline, de Tacite, de Sénèque, de Plutarque, a fourni à nos deux auteurs quatre «bonnes femmes » qui savent présenter à leurs maris chancelants l'image d'une mâle constance, rivalisant de résolution la masculinité tant vantée du héros typiquement romain, la surpassant même «en beauté et en gloire». Mais Shakespeare et Montaigne apportent tous les deux à leur lecture des textes antiques une sensibilité née autre part, dans le romantisme du moyen-âge tardif peut-être, producteur d'une littérature d'histoires amoureuses dont Shakespeare raffole, mais dont Montaigne affecte de se détourner la plupart du temps avec mépris. Cependant, nos deux auteurs se rencontrent sur le lieu de la vie conjugale des femmes choisies. Montaigne en souligne la tendresse par le moyen de phrases ajoutées et par son agencement des textes antérieurs, tandis que Shakespeare nous introduit en plein dans l'intimité du couple représenté sur la scène. Un autre point de rencontre serait l'attitude sceptique adoptée à l'égard des prétentions des «plus excellens hommes », soit Brutus, soit Alexandre, à laquelle seul Sénèque échappe en se pliant à la douceur d'être «cher à sa femme» pour devenir «plus cher à soi-même». En revanche, ce scepticisme ne semble pas toucher 
nos quatre «bonnes femmes », malgré le ton mordant dont Montaigne fustige la généralité des cas des femmes mariées ${ }^{8}$.

Le couple Antoine-Cléopâtre semble donner un tout autre tour à ce topos de la femme forte venue à l'appui de l'homme en lutte avec luimême. Antoine repousse ses deux épouses légitimes, Fulvie et Octavie, qui auraient pu le rétablir sur le terrain solide de l'honneur romain, pour succomber aux molles influences d'une égyptienne née sous le signe du serpent et de la liquidité. Le héros plongé dans l'érotisme se féminise, mais notre imagination se féminise aussi, séduite non par la femme, mais par les mots, dont l'enchantement nous berce avec délices dans l'oubli de l'action héroïque. Embarquée sur le Cydnus, Cléopâtre éclipse la Vénus peinte d'Apelles sortant des eaux, «O'er-picturing that Venus where we see The fancy outwork nature» (Antony and Cleopatra, II.ii.200-1). Ce triomphe de Cléopâtre est surtout un triomphe de l'art. Trois hommes parlent ensemble sur la scène. La femme en est absente. Elle est remplacée par les mots, mots pleins d'une sensualité en voûtante :

I will tell you.

The barge she sat in, like a burnish'd throne

Burn'd on the water : the poop was beaten gold.

Les mots sont empruntés pour la plupart à Plutarque, mais Plutarque ne fait qu'énumérer les choses ${ }^{9}$. Shakespeare leur donne une vie passionnelle en faisant du plus simple rapport entre deux objets une métaphore érotique. Les choses inanimées brûlent, elles battent, elles caressent,

Purple the sails, and so perfumed that

The winds were love-sick with them; the oars were silver,

Which to the tune of flutes kept stroke, and made

The water which they beat to follow faster,

As amorous of their strokes.

(II.ii.193-7)

\footnotetext{
${ }^{8}$ Pour les portraits de femmes dans les pièces shakespeariennes qui traitent de l'antiquité, on consultera, par exemple : R. A. Brower, Hero and Saint : Shakespeare and the GraecoRoman Muse (Oxford, Clarendon Press, 1971) ; C. Kahn, Roman Shakespeare: Warriors, Wounds, and Women (Londres et New York, Routledge, 1997); M. B. Rose, Gender and Heroism in Early Modern English Literature (Chicago et Londres, Chicago University Press, 2002).

${ }^{9}$ On trouvera le passage correspondant de Plutarque, dans la version de North utilisée par Shakespeare, dans l'appendice de l'édition citée, p. 246-7.
} 
Dans un passage célèbre du chapitre Sur des vers de Virgile (III, 5), Montaigne aura recours à Lucrèce pour dépeindre l'amour adultère de Vénus avec le dieu Mars. Mars, énervé, cède aux blandices de Vénus, «vaincu par la blessure éternelle de l'amour ${ }^{10}$. C'est le type même du héros excessivement amoureux, affaibli, féminisé, de même qu'Antoine dont les yeux « That o'er the files and musters of the war / Have glow'd like plated Mars » (I.i.2-4), est transformé dès les premiers vers de la pièce en captif asservi. Mais pour Montaigne, tout comme pour Shakespeare, ce sont les mots qui ont pouvoir de séduction, bien plus captivants que n'importe quelle femme ou déesse. Nos deux auteurs sont formés par l'enseignement des humanistes. Ils puisent dans le même fonds de culture, c'est-à-dire la littérature gréco-romaine qui leur fournit une thématique, une morale, et surtout des formes d'expression qui relèvent des préceptes de la rhétorique. Lorsque Shakespeare orne de métaphores la prose dénudée de Plutarque, il met en pratique les leçons de l'élocution (elocutio), la partie de la rhétorique consacrée au langage figuré. Lorsque Montaigne se penche sur Mars couché dans le giron de Vénus, c'est pour peser la force des mots utilisés par Lucrèce, sachant bien que l'analyse rhétorique du choix et de l'emplacement des mots peut rendre raison du pouvoir séducteur d'une «eloquence ... qui ne plaict pas tant comme elle remplit et ravit; et ravit le plus les plus fors espris » (Essais, p. 850).

Déjà dans le même chapitre, le «fort esprit » qu'est Montaigne s'est laissé volontairement séduire par Vénus présente seulement dans les mots: "Venus n'est pas si belle toute nue, et vive, et haletante, comme elle est icy chez Virgile»(p.826). Et Montaigne de citer quelques vers de Virgile qui évoquent l'étreinte douce et passionnée des époux, Vénus et Vulcain ${ }^{11}$. Il est vrai que Montaigne trouve cette Vénus «un peu bien esmue pour une Vénus maritale» (p. 827). Ce morceau s'insère dans un long développement sur le mariage, qu'il ne veut pas trop confondre avec l'amour. Ce n'est pas pourtant dans le but de déprécier le mariage. Au contraire, «ung bon mariage, s'il en est, refuse la compaignie et conditions de l'amour» (p. 829). C'est qu'il tâche de représenter celles de l'amitié, et l'amitié, comme Montaigne dit autre part, est bien supérieure à l'amour. Malheureusement, les femmes n'en sont pas capables, étant donné que leur âme n'a pas assez

${ }^{10}$ Lucrèce, De rerum natura, I, 33-40

${ }^{11}$ Virgile, Énéide, VIII, 387-92, 404-6. 
de fermeté ${ }^{12}$. L'idée du mariage d'amitié résiste à peine au scepticisme auquel échappent seules ses trois bonnes femmes. Elle y résiste quand même, et se caractérise par la «constance», la fermeté d’âme, la « fiance », et « un nombre infiny d'utiles et solides offices et obligations mutuelles» (p. 829). Les trois bonnes femmes sont celles qui ont donné l'exemple presque inouï d'un mariage qui ressemble à une amitié parfaite, encore plus parfaite que l'amitié masculine en raison de ce que le corps, aussi bien que l'âme, a part à l'alliance. C'est l'idéal que Montaigne juge impossible à réaliser dans De l'amitié (I, 28, 185) ; encore ne se voit-il qu'aux approches de la mort.

Encore plus surprenant que cette rare exemplarité du bon mariage chez Montaigne, serait le triomphe ultime du mariage dans Antony and Cleopatra. Antoine délaisse cruellement ses deux bonnes femmes romaines pour se perdre dans les sinuosités de l'Égypte. Mais sa fin sera romaine, inspirée par le faux bruit du suicide de Cléopâtre. Il ne se verra pas accusé

The courage of a woman, less noble mind

Than she which by her death our Caesar tells

'I am conqueror of myself'.

(IV.xiv.59-62)

Il mourra «a Roman, by a Roman Valiantly vanquish'd» (IV.xv.57-8). Encore plus romaine sera la mort de Cléopâtre :

We'll bury him : and then, what's brave, what's noble,

Let's do it after the high Roman fashion,

And make death proud to take us.

(IV.xv.86-8)

My resolution's plac'd, and I have nothing

Of woman in me : now from head to foot

I am marble-constant.

(V.ii.237-9)

Émule, non plus vainqueur, d'Antoine, elle ira encore une fois à sa rencontre, «I am again for Cydnus, To meet Mark Antony » (v.ii.2278 ), parée pour son dernier acte de séduction, dans laquelle Antoine l'a déjà devancée, lorsqu'il disait :

I will be

A bridegroom in my death, and run into't As to a lover's bed.

(IV.xiv.99-101)

12 Voir le chapitre De l'amitié, I, 28, p. 184-5. 
Mais c'est un mariage qu'ils vont consommer, Cléopâtre ayant bien mérité son titre d'épouse légitime :

Husband, I come :

Now to that name, my courage proves my title!

(V.ii.286-7)

Plutarque avait fourni à Shakespeare presque tous les détails du suicide de Cléopâtre, mais chez lui elle ne dit $\operatorname{mot}^{13}$. Chez Shakespeare elle est dotée de l'art de bien parler, pour nous plaire, pour nous émouvoir, pour nous en imposer à la fin en citant hautement ses vrais titres de noblesse.

Nous avons exploré quelques lieux de rencontre entre Montaigne et Shakespeare habités par les femmes sous le signe de l'amour et du mariage. Le théâtre de Shakespeare est largement ouvert aux femmes; les Essais, pour la plupart leur font un assez mauvais accueil. Pour en trouver de bonnes dans les pages de Montaigne, il faut le plus souvent aller de biais, les cherchant dans les exemples tirés de l'histoire romaine, dans les vers des poètes latins, et, enfin, dans son langage métaphorique. Dans les dernières pages du dernier chapitre, De l'expérience (III, 13), où Montaigne, aux approches de la mort, s'est fait l'ami de son corps dans la douleur comme dans la volupté, la métaphore du mariage se montre de plus en plus fréquente. Montaigne l'emploie pour désigner ce «très-convenable mariage du plaisir avec la necessité », de l'âme avec le corps : que l'âme «assiste et favorise [le corps] et ne refuse point de participer à ses naturels plaisirs et de s'y complaire conjugalement » (p. 1094, 1091). De cette façon, moyennant la rhétorique des locutions figurées, Montaigne intègre le bon mariage, celui qui reprend les conditions de l'amitié, dans son testament final, nous recommandant de «bien et naturellement sçavoir vivre cette vie » (p. 1091). On se croirait loin des dernières pièces de Shakespeare. On se souviendra pourtant que c'est en se mariant d'amour que les jeunes protagonistes dans ces pièces rachètent en quelque sorte les fautes de leurs aînés et que cette clôture se prépare dans des pays loin des centres civilisés, pays où l'on sait bien et naturellement vivre cette vie.

Ann Moss

Université de Durham

\footnotetext{
13 Voir l'annexe de l'édition citée, p. 277-8. Remarquons que la référence au mariage se fait chez Plutarque au moment du suicide d'Antoine (p. 273); Shakespeare la déplace magistralement.
} 